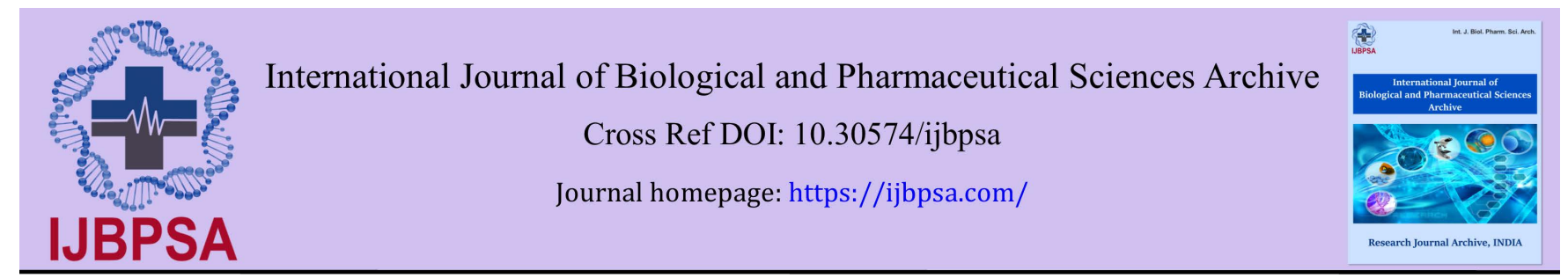

(RESEARCH ARTICLE)

\title{
Assessment of vitamin D among Sudanese Patients with Hypothyroidism
}

\author{
Hasna Osman Fadalla Mohamed ${ }^{1}$, Omer Fadol Edris ${ }^{2}$, Gad Allah Modawe ${ }^{3, *}$ and Suhair Abdelrahman \\ Ahmed 1 \\ ${ }^{1}$ Department of Clinical Chemistry, Faculty of Medical Laboratory Science, Al Neelain University, Khartoum, Sudan. \\ 2 Department of Biochemistry, Faculty of Sciences and Technology, Al Neelain University, Khartoum, Sudan. \\ ${ }^{3}$ Department of Biochemistry, Faculty of Medicine, Omdurman Islamic University, Omdurman, Sudan.
}

International Journal of Biological and Pharmaceutical Sciences Archive, 2021, 01(02), 057-061

Publication history: Received on 28 January 2021; revised on 01 March 2021; accepted on 04 March 2021

Article DOI: https://doi.org/10.30574/ijbpsa.2021.1.2.0024

\begin{abstract}
Background: Vitamin D deficiency is a worldwide health issue and its role as an immune modulator has recently been emphasized. Evidence increasingly indicates the important role of vitamin D in reducing the incidence of autoimmune diseases. However, at this time the search for its role in autoimmune diseases and thyroid is not critical.
\end{abstract}

Objectives: The study aimed to assess the relationship between vitamin D deficiency in Sudanese patients with hypothyroidism.

Methods: Analytical case control hospital based study, conducted in Fadil Hospital and Police Hospital, Khartoum city, Khartoum state. The study carried out from March 2018 to September 2018. A total of 100 subjects were enrolled in the study. Vitamin D deficiency was set at levels below $20 \mathrm{ng} / \mathrm{ml}$. Thyroid hormones (TSH, T3 and T4) were assessed in all participants using auto analyzer TOSOH AIA 313,while vitamin D was estimated using ELIZA. The data were analyzed using SPSS version (21).

Results: The study included $76(76 \%)$ females and $24(24 \%)$ males, no significance differences between thyroid profile among study population.75 (75\%) deficiency of vitamin D and 25(25\%) normal vitamin D. vitamin D deficiency was significantly lower in female than male with $(p=0.001)$.

Conclusion: The results indicated that patients with hypothyroidism suffer from vitamin D deficiency significantly associated with the degree and severity of hypothyroidism. This encourages the desirability of vitamin D supplementation and recommends the detection of vitamin D deficiency for all hypothyroidism patients.

Keywords: Vitamin D deficiency; Thyroid disease, hypothyroidism

\section{Introduction}

Vitamin D deficiency is a global health problem and over a billion people worldwide are vitamin D deficient or insufficient [1] Yet no international health organization or governmental body has declared a health emergency to warn the public about the urgent need of achieving sufficient vitamin D blood levels [2]. Understanding of the role of vitamin D has been evolving since its discovery in the early 20th century from being a simple vitamin to a steroid pro-hormone, it has been recognized to be involved in various immune functions as well as bone and muscle development [3]

\footnotetext{
${ }^{*}$ Corresponding author: Dr. Suhair Abdelrahman Ahmed

Department of Clinical Chemistry, Faculty of Medical Laboratory Science, Al Neelain University, Khartoum, Sudan. 
Vitamin D deficiency has been shown to be associated with autoimmune diseases, including rheumatoid arthritis (RA), systemic lupus erythematosus (SLE), inflammatory bowel disease (IBD), multiple sclerosis (MS) and type 1 diabetes (T1DM), and that vitamin D supplementation prevents the onset and/or development of these autoimmune diseases [4]. Furthermore, it was reported that patients with Hashimoto's thyroiditis, an autoimmune thyroid disease had lower vitamin D levels [5]. Vitamin D plays an essential role in calcium homeostasis and the development and maintenance of the skeleton [6]. It is recognized as the sunshine fat-soluble vitamin. Exposure to ultraviolet light (290-320 nm) are the main source of vitamin D [7]. In the classical endocrine pathway, vitamin D enters the circulation attached to a D-binding protein, is first hydroxylated in the liver to $25(\mathrm{OH}) \mathrm{D}$ and then in the kidney to form the active metabolite, 1,25 dihydroxy vitamin D $(1,25-(\mathrm{OH}) 2 \mathrm{D})$ or calcitriol [8]. Serum $25(\mathrm{OH}) \mathrm{D}$, the most abundant circulating precursor of active vitamin $\mathrm{D}$, is the most widely accepted indicator of vitamin $\mathrm{D}$ status and reflects combined contributions from cutaneous synthesis. Serum 25(OH)D has a half-life of approximately two to three weeks, in contrast, 1,25-(OH)2D has a short circulating half-life and is tightly regulated over a narrow range by parathyroid hormone, calcium and phosphate [9]. Serum $1,25-(\mathrm{OH}) 2 \mathrm{D}$ is not a good measure of vitamin D status since a decrease may not occur until vitamin D deficiency is severe [10]. Levels of 25(OH)D 30 to $32 \mathrm{ng} / \mathrm{ml}$ is considered to be sufficient, but levels of 20 to $29 \mathrm{ng} / \mathrm{mL}$ is insufficient and if it is less than $12 \mathrm{ng} / \mathrm{ml}$ is considered an evidence of severe vitamin D deficiency [11]. Importantly, both vitamin $\mathrm{D}$ and thyroid hormone bind to similar receptors called steroid hormone receptors. A different gene in the Vitamin D receptor was shown to predispose people to autoimmune thyroid disease including Graves' disease and Hashimoto's thyroiditis. For these reasons, it is important for patients with thyroid problems to understand how the vitamin D system works. Vitamin D mediates its effect though binding to vitamin D receptor (VDR), and activation of VDRresponsive genes. While VDR gene polymorphism was found to associate with autoimmune thyroid diseases (AITDs) [12].

The study aimed to assess the relationship between vitamin D deficiency in Sudanese patients with hypothyroidism.

\section{Material and methods}

Analytical case control hospital based study, conducted in Fadil Hospital and Police Hospital, Khartoum city, Khartoum state. The study carried out from March 2018 to September 2018. A total of 100 subjects were enrolled in the study. Vitamin D deficiency was set at levels below $20 \mathrm{ng} / \mathrm{ml}$. Thyroid hormones (TSH, T3 and T4) were assessed in all participants using auto analyzer TOSOH AIA 313,while vitamin D was estimated using ELIZA. The data were analyzed using SPSS version (21). The study approved from ethical committee, Al Neelain University, faculty of medical laboratory sciences.

\subsection{Inclusion and exclusion criteria}

Patients with hypothyroidism were included in this study. All patients' taking supplements of vitamin D and diary product excluded from this study.

\section{Results}

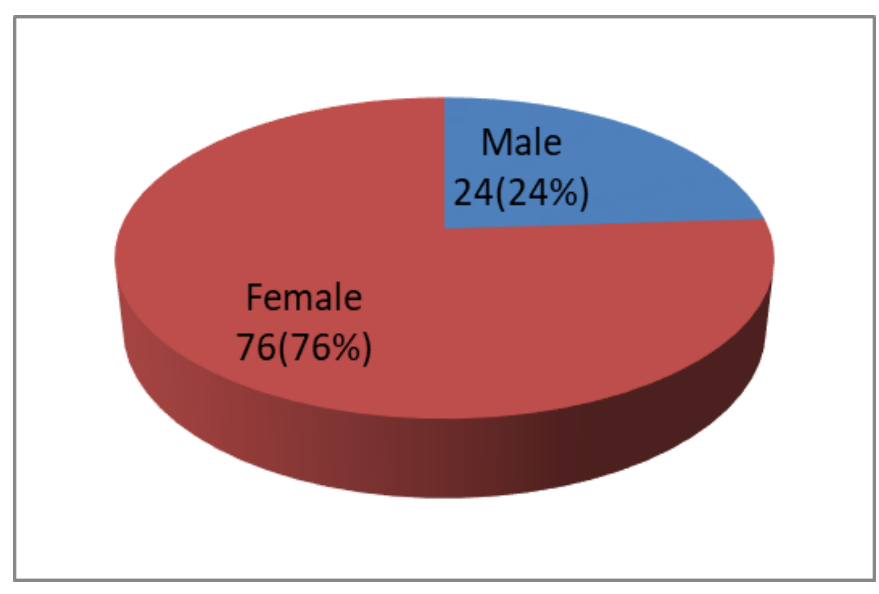

Figure 1 Distribution of patients according to gender. 


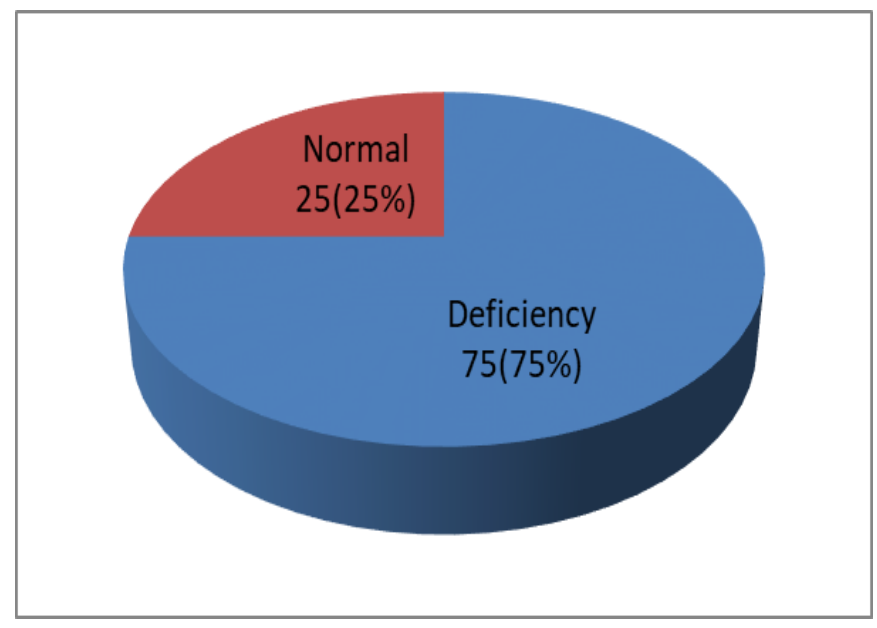

Figure 2 The percentage of vitamin D deficiency in patients with hypothyroidism

Table 1 mean comparison of thyroid hormones across vitamin D status

\begin{tabular}{|l|l|l|l|}
\hline Parameters & Deficiency $($ Mean \pm SD) & Normal $($ Mean \pm SD) & $P$-value \\
\hline FT3 & $4.67 \pm 0.82$ & $4.65 \pm 0.88$ & 0.936 \\
\hline FT4 & $14.97 \pm 2.56$ & $14.81 \pm 2.64$ & 0.772 \\
\hline TSH & $2.04 \pm 1.53$ & $1.59 \pm 1.25$ & 0.248 \\
\hline
\end{tabular}

Table 2 mean comparison of study parameters across the gender

\begin{tabular}{|l|l|l|l|}
\hline Parameters & Male $(M e a n \pm$ SD) & Female (Mean \pm SD) & $P$-value \\
\hline vit D & $29.39 \pm 15.37$ & $19.31 \pm 12.01$ & 0.001 \\
\hline FT3 & $4.51 \pm 0.69$ & $4.71 \pm 0.87$ & 0.235 \\
\hline FT4 & $14.59 \pm 1.91$ & $15.03 \pm 2.74$ & 0.379 \\
\hline TSH & $1.57 \pm 1.10$ & $2.04 \pm 2.54$ & 0.202 \\
\hline
\end{tabular}

Table 3 risk of vitamin D deficiency in female and male patients

\begin{tabular}{|l|l|l|l|l|l|}
\hline \multirow{2}{*}{ Gender } & \multicolumn{2}{|l|}{ vit D } & \multirow{2}{*}{ P-value } & OR & CI Lower-CI Upper \\
\cline { 2 - 3 } & Deficiency & Normal & & & \\
\hline Female & $64(85.3 \%)$ & $12(48.0 \%)$ & 0.000 & 6.303 & $(2.290-17.346)$ \\
\hline Male & $11(14.7 \%)$ & $13(52.0 \%)$ & & & \\
\hline
\end{tabular}

\section{Discussion}

Vitamin D is known for its essential role in the balance of bones and minerals. Interestingly, it has recently been shown that vitamin $\mathrm{D}$ has strong immune effects and plays important roles in the pathogenesis of autoimmune diseases [13].Serum concentration of $25(\mathrm{OH}) \mathrm{D}$ is the best indicator of vitamin D status. Vitamin D reflects the skin produced from food and supplements and has a long shelf life of up to 15 days. In contrast to $25(\mathrm{OH}) \mathrm{D}$, circulating $1,25(\mathrm{OH})_{2} \mathrm{D}$ is not a good indicator of vitamin D status due to a short half-life of 15 hours and serum concentrations are closely regulated by thyroid hormones, calcium and phosphate [14].Levels of $1,25(\mathrm{OH})_{2} \mathrm{D}$ do not usually decrease until severe vitamin D deficiency. Therefore, in this study we measured serum $25(\mathrm{OH}) \mathrm{D}$ instead of $1,25(\mathrm{OH})_{2} \mathrm{D}$ to ensure more accurate results. Few studies have been conducted in order to find any significant association between vitamin D levels and thyroid and determine whether vitamin D deficiency involves causing hypothyroidism or as a result of disease and those that have produced conflicting results. Some researchers have examined the prevalence of vitamin D deficiency 
in many countries, but this was one of a few studies aimed at evaluating vitamin D and its effect on hypothyroidism in Sudan, specifically in Khartoum state. We therefore performed this study to assess vitamin D levels among hypothyroid patients compared with health controls who did not complain of hypothyroidism or any thyroid disease. The results revealed lower levels of vitamin $D$ in females than males, otherwise this decrease is not important but we can refer this unimportant decrease to a small sample size from our study. In conformity with our results, previous studies have observed that serum levels $25(\mathrm{OH})$ D vary significantly between males and females. Moreover, Hashimpur et al. studied the prevalence of vitamin D in Tehran and found insignificant differences between males and females without an association between vitamin D and sun exposure [15] these results were contrary to our results.

Furthermore, the current study showed that vitamin D levels were significantly lower in hypothyroid patients compared with controls. The limitations of this study could be summarized in three points; first, the small number of subjects, second, limited in its ability to conclude that vitamin D status is directly related to the pathogenesis of hypothyroidism; third, we have to measure the parathyroid hormone (PTH) and clarify its effect on vit D and hypothyroidism disease. Therefore, the direct role of vitamin $D$ in those patients with thyroid problem should be examined by further prospective clinical studies and examine the effect of the treatment of vitamin D and PTH on hypothyroidism. Further studies with a larger number of subjects are needed to determine whether vitamin D deficiency is a casual factor in the pathogenesis of hypothyroidism or rather a consequence of the disease. Moreover, supplementary Vit D and calcium are recommended to patients with hypothyroidism

\section{Conclusion}

Our results indicated that patients with hypothyroidism suffered from hypovitaminosis D. Moreover, the positive significant correlation between each of serum vit D and calcium with thyroid hormones and that negative significant correlation with TSH levels, suggested that deficiency of serum vit D and calcium levels were significantly associated with degree and severity of the hypothyroidism which encourage the advisability of vit D supplementation. Screening for Vitamin D deficiency and serum calcium levels recommended for all hypothyroid patients.

\section{Compliance with ethical standards}

\section{Acknowledgments}

Our appreciation and gratefulness are extended to the patients from Fadil Hospital and Police Hospital who agrred to contribute in this study.

\section{Disclosure of conflict of interest}

No financial support and no other potential conflict of interest relevant to this work were reported.

\section{Statement of informed consent}

A written informed consent was obtained from all individual participants included in the study.

\section{References}

[1] Hollick MF, Chen TC. Vitamin D deficiency a worldwide problem with health consequences.Am J ClinNutr. 2008.

[2] Naeem Z. Vitamin D Deficiency- An Ignored Epidemic.Int J Health Sci (Qassim). 2010.

[3] Deluca HF. Evolution of our understanding of vitamin D. Nutr Rev. 2008.

[4] Baeke F, Takiishi T, Korf H, Gysemans C, Mathieu C, Vitamin D. modulator of the immune system. Curr.OpinPharmacol. 2010.

[5] Tamer G, Arik S, Tamer I, Coksert D. Relative vitamin D insufficiency in Hashimoto's thyroiditis. Thyroid. 2011.

[6] Cranney A, Horsley T, O'Donnell S. Effectiveness and Safety of Vitamin D in Relation to Bone Health. Evidence Reports/Technology Assessments. 2008.

[7] Michael FH. Sunlight and vitamin D for bone health and prevention of autoimmune diseases, cancers, and cardiovascular disease.Am J ClinNutr. 2004. 
[8] Qamar J, Khan MD, Carol J, Fabian MD. How I Treat Vitamin D Deficiency. J OncolPract. 2010.

[9] Friedman Theodore C. Vitamin D Deficiency and Thyroid Disease. www.goodhormonehealth.com/VitaminD.

[10] Kaplan MM. Clinical perspectives in the diagnosis of thyroid disease. Clin Chem. 1999.

[11] Vilarrasa N, Vendrell J, Maravall J, Elıo I, Solano E, San Jose E. Is plasma 25(OH) D related to adipokines, inflammatory cytokines and insulin resistance in both a healthy and morbidly obese population? Endocrine. 2010.

[12] Wolpowitz D, Gilchrest BA. The vitamin D questions: How much do you need and how should you get it? J Am AcadDermatol. 2006.

[13] Elsammak MY, Al-Wossaibi AA, Al-Howeish A, Alsaeed J. High prevalence of vitamin D deficiency in the sunny Eastern region of Saudi Arabia: a hospital-based study. East Mediterr Health J. 2011.

[14] Hashemipour S, Larijani B, Adibi H, Ebrahim J, Mojtaba S, Mohammad P. Vitamin D deficiency and causative factors in the population of Tehran. BMC. 2004.

[15] Sedrani SH. Low 25-hydroxyvitamin D and normal serum calcium concentrations in Saudi Arabia: Riyadh region. Ann NutrMetab. 1994. 